\title{
Needle-knife polypectomy in the duodenum assisted by peristaltic tension
}
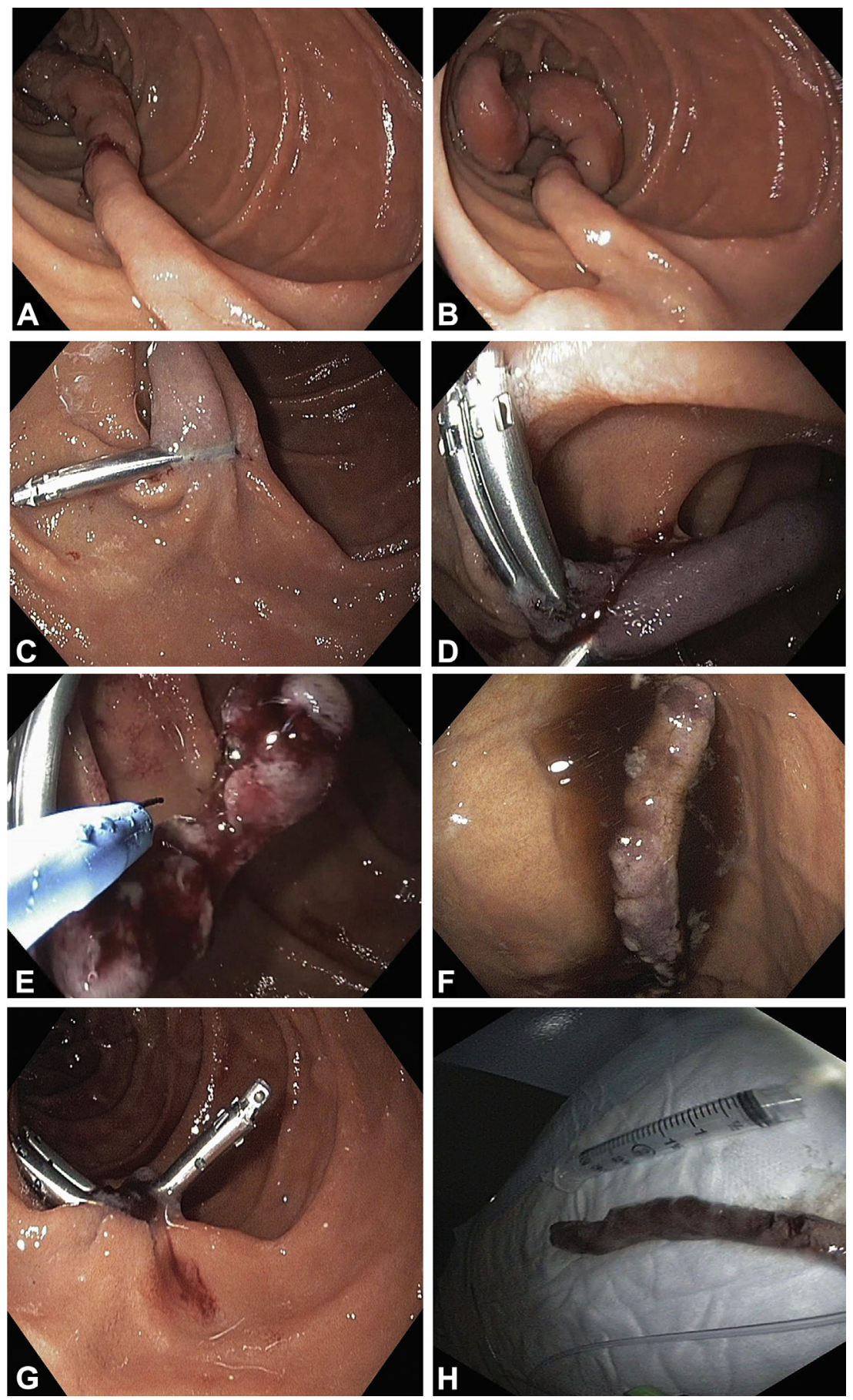

Figure 1. Stepwise management of a pedunculated duodenal polyp using a needle knife. The pedunculated duodenal polyp was not amenable to endoscopic snaring $(\mathbf{A}, \mathbf{B})$. Hemostasis clips were prophylactically applied $(\mathbf{C}, \mathbf{D})$ and the stalk was transected using a needle knife $(\mathbf{E})$. To prevent specimen loss, the polyp was withdrawn into the stomach $(\mathbf{F})$. Inspection of the polypectomy site revealed no hemorrhage or iatrogenic injury $(\mathbf{G})$. The retrieved specimen measured over $8 \mathrm{~cm}$ in maximum diameter $(\mathbf{H})$.

Written transcript of the video audio is available online at www.VideoGIE.org. 
A 46-year-old woman was referred for investigation of iron-deficiency anemia. History, examination, and laboratory investigation did not establish the cause. Fecal immunohistochemical testing was positive, and endoscopic evaluation was recommended (Video 1). EGD revealed a 0-1p polyp arising from the inferolateral wall of the second portion of the duodenum (Figs. $1 \mathrm{~A}$ and B). Snaring the lesion proved impossible despite repositioning attempts. Hemostasis clips were applied prophylactically to the polyp stalk (Figs. 1C and D). The stalk was then transected with a needle-knife (Fig. 1E), and the polyp was deposited into the gastric lumen to prevent loss of the specimen (Fig. 1F). Re-evaluation of the polypectomy site demonstrated no iatrogenic trauma or postpolypectomy hemorrhage (Fig. 1G). The specimen was then safely withdrawn from the patient (Fig. 1H). Histologic analysis demonstrated a fibroepithelial polyp with Brunner's gland hyperplasia. Pedunculated Brunner's gland adenomas arising from the second portion of the duodenum are rare. These hamartomatous polyps rarely become malignant and are usually asymptomatic. GI bleeding or obstruction may necessitate resection. Snare polypectomy of pedunculated polyps in the duodenum can be difficult because of distal migration of the lesion and angulation of the bowel. If used with caution, needle-knife transection facilitates polypectomy. In this case, safe transection was aided by peristaltic tension placed on the stalk. The greatest risk with this technique is inadvertent thermal damage to the bowel wall, perforation, or both. A ball-tip or hooktip needle-knife may reduce this risk.

\section{DISCLOSURE}

All authors disclosed no financial relationships relevant to this publication.

David Prichard, MB, PhD, Division of Gastroenterology, Mayo Clinic Health System La Crosse, La Crosse, Wisconsin, USA, Fergal Donnellan, MB, MD, Division of Gastroenterology, Vancouver General Hospital, University of British Columbia, Vancouver, British Columbia, Canada

Copyright (c) 2016 The Authors. Published by Elsevier, Inc. on behalf of the American Society for Gastrointestinal Endoscopy. This is an open access article under the CC BY-NC-ND license (http://creativecommons.org/ licenses/by-nc-nd/4.0/).

http://dx.doi.org/10.1016/j.vgie.2016.07.003 